\title{
INVERSION OF ABELIAN INTEGRALS ${ }^{1}$
}

\author{
BY GEORGE KEMPF ${ }^{2}$
}

0. Introduction. There are naturally occurring bundles over the Jacobian variety of a compact Riemann surface. These Picard bundles are involved in an essential way in many global problems about the Jacobian. Unfortunately, we have no good description of these bundles other than an equivalent formulation of their definition. Their nonabelian nature seems to present a major obstacle to giving a rigid description of them. The problem of finding an alternate description of these Picard bundles is what I call an inversion problem.

My main objective in this paper is to describe some possible ways of solving the inversion problem and report on the partial results in these directions. Some interesting properties of the Picard bundles will also be indicated. Further research on these inversion problems is clearly required. Their solutions would lead to a more explicit theory of Riemann surfaces. Even good methods of finessing their solutions would be an important advance in this fundamental region of algebraic geometry.

1. Abelian integrals. Let $S$ be a compact Riemann surface which has genus g. Topologically $S$ is just the surface of a doughnut with $g$ holes. The cohomology group $H^{1}(S, Z)$ is a free Abelian group of rank $2 g$, which has a unimodular alternating pairing given by intersection.

Let $\omega$ be an Abelian differential on $S$. We may regard $\omega$ as a global section of the sheaf $\Omega_{s}$ of holomorphic one-forms on $S$. Thus $\omega$ locally has the form $d f$, where $f$ is a holomorphic function. Globally the indefinite integral $\int \omega$ is a multivalued function, which is locally holomorphic and only determined up to adding a constant of integration.

Usually one analyzes the multivaluedness of these integrals as follows. Let $t$ be a fixed point on $S$. For any path $\sigma$ from $t$ to a variable point $s$, the definite integral $\int_{\sigma} \omega$ is locally a holomorphic function of its endpoint $s$ and is a linear function of $\omega$. Furthermore, for any other choice of the path, say $\tau$, we have $\int_{\tau} \omega=\int_{\sigma} \omega+\int_{\tau \circ \sigma^{-1}} \omega$, where $\int_{\tau \circ \sigma^{-1}} \omega$ is called the period of $\omega$ around the closed path $\tau \circ \sigma^{-1}$. As linear functionals, we have

$$
\int_{\tau}=\int_{\sigma}+\int_{\text {closed path }}
$$

Received by the editor February 15, 1980.

1980 Mathematics Subject Classification. Primary 14H05, 14H40, 30F10; Secondary 14K20, 32G15, 14F05.

${ }^{1}$ Based on the author's address to the Society at the New York meeting, April 1979.

${ }^{2}$ Partly supported by NSF Grant \# 7900965. 
To eliminate the multivaluedness of the integral $\int_{t}^{s} \omega$, which is due to the choice of path from $t$ to $s$, one interprets this expression as defining an analytic mapping,

$$
\int_{t}: S \rightarrow \Gamma\left(S, \Omega_{S}\right)^{\text {dual }} / L=J
$$

where $L$ is the subgroup of linear functionals on the space of abelian differentials, which are gotten by integrating around closed paths in $S$. The space $V \equiv \Gamma\left(S, \Omega_{S}\right)^{\text {dual }}$ is a complex vector space of dimension $g$. The subgroup $L$ is a lattice in $V$. It is called the period lattice and is naturally isomorphic to $H^{1}(S, \mathbf{Z})$. The quotient $J=V / L$ is called the Jacobian variety. It is a compact analytic group of dimension $g$.

Similarly, for any positive integer $i$, the Abelian sum

$$
\int_{t}^{s_{1}} \omega+\cdots+\int_{t}^{s_{i}} \omega \equiv \int_{i t}\left(s_{1}+\cdots+s_{i}\right)(\omega)
$$

defines an analytic mapping

$$
\int_{i t}: S^{(i)} \equiv(S \times \underset{i \text { times }}{\ldots} \times S) / / \text { permutations of the factors } \rightarrow J,
$$

where $S^{(i)}$ is a complex manifold of dimension $i$, which is called the $i$ th symmetric of $S$.

For the remainder of this paper, I will require that $i>2 g-2=$ degree $\left(\Omega_{S}\right)$. In this case, the Abelian sum mapping has a very remarkable property.

THEOREM 1. The integral $\int_{i t}: S^{(i)} \rightarrow J$ is a locally trivial bundle of projective spaces $\mathbf{P}^{i-g}$.

In the classical theory one may easily write such local trivializations via Riemann's theta function. The global problem of describing how this bundle is twisted up will be called an inversion problem. These problems naturally arise when one tries in some sense to invert the Abelian sum mapping. They arise as part of the general philosophy of inversion where one tries to control aspects of the Riemann surface in terms of the Jacobian with some auxiliary structures. This philosophy of inversion was decisively applied by Abel when $g=1$ and one would like to have a better inversion theory when $g \geqslant 2$.

2. An analytic inversion problem. This type of inversion problem asks whether one may imitate the procedure which Riemann used to describe the theta divisor on the Jacobian $J$ by finding a theta function on the covering space $V$. In his case this theta function is determined up to constant multiple by the multipliers $\left\{e_{l}\right\}$ in the functional equations, $\theta(v+l)=e_{l}(v) \theta(v)$ for $l$ in $L$, which it satisfies. In Riemann's case, these multipliers are much more elementary functions than $\theta$ itself. Next $I$ will explain in some detail how a solution to this analytic inversion problem should look.

Given any bundle $s: T \rightarrow J$ over the Jacobian, we may form the induced bundle $\tilde{s}: \tilde{T} \rightarrow V$ over the universal covering space $V$ of $J$. Furthermore the lattice $L$ acts naturally on $\tilde{T}$ such that $\tilde{s}$ is $L$-equivariant and the induced 
mapping $\tilde{s} / L: \tilde{T} / L \rightarrow V / L$ is naturally identified with $s$. Explicitly, if $\pi$ : $V \rightarrow J$ is the quotient homomorphism, $\tilde{T}=\{(t, v) \in T \times V \mid s(t)=\pi(v)\}$, $\tilde{s}(t, v)=v$ and $l *(t, v)=(t, l+v)$ for $l$ in $L$. Thus to describe a bundle over $J$ we have to give an $L$-equivariant bundle over $V$.

The simplest $L$-equivariant bundles $\tilde{s}: \tilde{T} \rightarrow V$ are trivial as bundles but have an interesting $L$-action. In this case, we have an isomorphism (or trivialization) $\rho: F \times V \underset{\rightarrow}{\approx} \tilde{T}$ such that $\tilde{s}(\rho(f, v))=v$ for all $(f, v)$ in $F \times V$. In terms of this trivialization, the $L$-action on $\tilde{T}$ has the form

$$
l *(f, v)=\left(g_{l}(f, v), v+l\right) \text { for }(l, f, v) \text { in } L \times F \times V,
$$

where the mapping $g_{l}: F \times V \rightarrow F$ gives an isomorphism $e_{l}(v)$ of the fiber $F$ for each $l$ and $v$. Thus our action is given by

$$
l *(f, v)=\left(e_{l}(v)(f), v+l\right) .
$$

For these multipliers $e_{l}(V)$ to give a group action, they must satisfy the identities,

$$
e_{l^{\prime}+l}(v)=e_{l^{\prime}}(v+l) \circ e_{l}(v) \text { for all } l \text { and } l^{\prime} \text { in } L,
$$

where the circle $\circ$ denotes the composition of automorphisms of $f$. A system of multipliers $\left\{e_{l}(v)\right\}$ satisfying $(* *)$ are called factors of automorphy.

Now, if we use the fact that $L$ is a free abelian group with generators $l_{1}, \ldots, l_{2 g}$, the above $L$-action is determined by the transformations of $\tilde{T}$ given by the generators subject to the sole condition that these transformations commute. In terms of multipliers, let $f_{i}(v) \equiv e_{l_{i}}(v)$ for $1 \leqslant i \leqslant 2 g$. These special multipliers satisfy the commutativity conditions,

$$
f_{i}\left(v+l_{j}\right) \circ f_{j}(v)=f_{j}\left(v+l_{i}\right) \circ f_{i}(v) \text { for } 1 \leqslant i<j \leqslant 2 g .
$$

Conversely, given such $2 g$ multipliers satisfying these $g(2 g-1)$ equations $(* * *)$, one may easily recover all the multipliers $e_{l}(v)$ by using equation (**) and these $e_{l}(v)$ will be factors of automorphy.

With these general remarks out of the way, we can state

THEOREM 1. The bundle $\int_{i t}: S^{(i)} \rightarrow J$ of $\mathbf{P}^{i-g}$ 's induces a complex-analytically trivial bundle over $V$. Hence it can be described by factors of automorphy $\left\{e_{l}(v) \mid l \in L\right\}$, which are complex analytic functions on $V$ with values in the projective general linear group, Aut $\left(\mathbf{P}^{i-g}\right)$.

This theorem [2, 4] may be proven using general methods of Grauert, but there is no known way to construct such a trivialization explicitly.

Problem. Pick out some particular trivialization and compute the multipliers.

To find such a trivialization one would like to restrict one's choice. One possible condition, that one could require, is a variation on one of Riemann's themes. Assume that $l_{1}, \ldots, l_{2 g}$ is a basis of $L$ such that the intersection numbers $\left[l_{i}: l_{j}\right]=0$ unless $i+j=2 g+1$. As $l_{1}, \ldots, l_{g}$ are a complex basis for $V$, we may identify $V$ with $\mathbf{C}^{g}$ so that $l_{1}=(1,0, \ldots, 0), \ldots, l_{g}=$ $(0, \ldots, 0,1)$. 
With this notation, the possible condition is contained in

THEOREM 2. We may choose the trivialization of Theorem 1 so that the multipliers $f_{i}(v)$ are the identity for $g \leqslant i \leqslant 2 g$.

The proof $[5,3]$ of this theorem uses the periods of Prym differentials and it gives an explicit trivialization in some neighborhood of $\mathbf{R}^{g}$ in $\mathbf{C}^{g}$.

If one uses a trivialization as in Theorem 2 , the bundle $S^{(i)} \rightarrow J$ is determined by the $g$ multipliers $f_{1}(\underline{x}), \ldots, f_{g}(\underline{x})$ subject only to the conditions:

(a) they are entire functions on $\mathbf{C}^{g}$ with values in $\operatorname{Aut}\left(\mathbf{P}^{i-g}\right)$;

(b) they are $g$-tuplely periodic with respect to the integral vectors in $\mathbf{C}^{\mathbf{g}}$; and

(c) they satisfy the $g(g-1) / 2$ commutativity relations $(* * *)$ for $1 \leqslant i<j$ $\leqslant g$.

REMARK 1. The simplest unknown case is when $g=2$ and $i=3$. In this case, one wants to find two doubly periodic functions $f_{1}$ and $f_{2}$ on $\mathbf{C}^{2}$ with values in $\operatorname{Aut}\left(\mathbf{P}^{1}\right)$ satisfying only one commutativity relation. Even here I have had trouble finding nontrivial solutions to this relation.

REMARK 2. The above desired trivializations depend on the choice of a canonical basis of the lattice $L$. One may hope to have some simple rule for passing between such trivializations for two different choices of canonical bases. Furthermore one would like to understand the dependence of such trivializations on the choice of the "fixed" point $t$ of $S$.

3. Linearization of the inversion problem. Instead of working with the projective bundles $\int_{i t}: S^{(i)} \rightarrow J$ given by integration, one constructs complex analytic vector bundles $\mathbf{W}_{i} \rightarrow J$ over the Jacobian so that $\int_{i t}$ is the projective bundle of lines in the fibers of $\mathbf{W}_{i}$. The vector bundles $\mathbf{W}_{i}$ are called Picard bundles.

The Picard bundles may be described by giving the sheaf $\mho_{i}$ of the analytic sections of $\mathbf{W}_{i}$. These Picard Sheaves $\mathcal{W}_{i}$ are locally free $\hat{\theta}_{J}$-modules of rank $i-g+1$ on the Jacobian $J$. Explicitly, one may define $W_{i}$ as the dual sheaf to the direct image sheaf $\left(\int_{i t}\right)_{*}\left[\Theta_{S^{(i)}}\left(S^{(i-1)}+t\right)\right]$ on $J$, where $S^{(i-1)}+t$ is the divisor on $S^{(i)}$ which consists of divisors on $S$ containing the fixed point $t$. This construction works because $S^{(i-1)}+t$ intersects a fiber of $\int_{i t}$ in a hyperplane section. One should note that the dual Picard bundle $\mathbf{W}_{i}^{*}$ has a canonical section corresponding to $\left(\int_{i t}\right)_{*}$ (constant function 1 ).

In this context, we may ask for a solution of the linearized inversion

Problem. Describe the Picard bundles $\mathbf{W}_{i} \rightarrow J$ by factors of automorphy in $\mathbf{G} L(i-g+1, \mathbf{C})=\operatorname{Aut}\left(\mathbf{A}^{i-g+1}\right)$.

In this situation the analogous versions of Theorems 1 and 2 still hold. In this linear version, the multipliers $f_{1}(\underline{x}), \ldots, f_{g}(\underline{x})$ are matrices whose coefficients are entire functions. One may hope to choose them as to satisfy the additional conditions:

(d) the coefficients of the multipliers are first order entire functions on $V$; and

(e) the canonical section of $\mathbf{W}_{i}^{*}$ in terms of the trivialization is given by a $(i-g+1)$-vector of first order entire functions. 
In the 1960's, A. Mattuck $[10,11]$ studied how the bundles $\int_{i t}: S^{(i)} \rightarrow J$ were twisted up. His most significant results were the determination of the Chern classes of Picard sheaves $w_{i}$ in the Chow ring of rational equivalence classes of cycles on $J$. Also Schwarzenberger [16] and Macdonald [9] made contributions to this subject. During this period the subject became interlaced with Grothendieck's general theory of Picard schemes [1, 12], but the basic inversion problems about curves were somewhat ignored. In the rest of this section, I want to give a sample of the above mathematicians' viewpoints.

First recall that, by Abel's theorem and its converse, the fibers of the Abelian integral $\int_{i t}: S^{(i)} \rightarrow J$ are complete linear systems of divisors. Thus, for two effective divisors $D_{1}$ and $D_{2}$ on $S$ of degree $i, \int_{i t}\left(D_{1}\right)=\int_{i t}\left(D_{2}\right)$ if and only if the invertible sheaves $\theta_{S}\left(D_{1}\right)$ and $\theta_{S}\left(D_{2}\right)$ on $S$ are isomorphic. In other words, for each point $j$ of $J$, there is an invertible sheaf $\mathcal{L}_{j}$ on $S$ such that the fiber $\left(\int_{i t}\right)^{-1}(j)$ is isomorphic to the projective space of lines in the vector space $\Gamma\left(S, \mathcal{L}_{j}\right)$.

Globally we may form an invertible sheaf $\mathcal{E}$ on the product $S \times J$ such that, for each point $j$ of $J,\left.\mathcal{L}\right|_{S \times j}$ is isomorphic to the previous sheaf $\mathcal{E}_{j}$. This sheaf $\mathcal{L}$ is uniquely determined by this provided we require an isomorphism $\left.\mathcal{L}\right|_{t \times J} \approx \theta_{J}$. Classically Riemann could easily describe $\mathcal{E}$ using his theta function. In Grothendieck's rendition, $\mathcal{E}$ is a family of invertible sheaves of degree $i$ on $S$ parameterized by $J$, which is characterized by a universal mapping property.

The global version of Abel's theorem and its converse may now be simply stated as

THEOREM 3. The Picard sheaf $\mathscr{W}_{i}$ is isomorphic to the direct image $\left(\operatorname{proj}_{J}\right)_{*} \mathfrak{L}$ of $\mathcal{L}$ by the projection of $S \times J$ onto $J$.

One possible use of this theorem is that it gives a direct relationship between global properties of the Picard sheaf $\mathscr{W}_{i}$ and those of the sheaf $\mathcal{L}$ on the product $S \times J$. The main advantage is that one may study global properties of $\mathcal{L}$ by means of its projection on the other factor $S$. This approach is a variation on the theme entitled "divisorial correspondences".

Lastly I want to mention the most accessible special case of Mattuck's results concerning the Chern classes of Picard sheaves. This particular result is equivalent to the determination of their first Chern class.

THEOREM 4. The invertible sheaf $\Lambda^{i-g+1} W_{i}$ is isomorphic to $\theta_{J}(-\theta)$, where the theta divisor $\theta$ is the image of the Abelian integral $\int_{(g-1) t}: S^{(8-1)} \rightarrow J$.

This result shows how the above inversion problems are direct generalizations of the problem which Riemann solved in determining the factors of automorphy in the functional equation for the theta function. In fact, the functional equation of the theta function describes how the sheaf $\theta_{J}(-\theta)$ is twisted up.

4. Encouraging results. When one contemplates the linear inversion problem, one realizes that one is required to pick out a particular locally free sheaf 
$\mathscr{Q} S_{i}$ on the Jacobian variety $J$. To do this, one would have to be able to distinguish a Picard sheaf $\mho_{i}$ from all nearby locally free sheaves.

For most curves, I was able to show the encouraging result that any sheaf near to $\mathscr{W}_{i}$ is a trivial modification of $\mho_{i}$ itself. Explicitly, consider the obvious family of locally free sheaves on $J$,

$$
\mathcal{T}\left(\mathcal{W}_{i}\right)=(\text { translation by } j) * \mathcal{W}_{i} \otimes(\text { invertible sheaf on } J)
$$

where $j$ is a point of $J$. This gives a family of locally free sheaves on $J$ parameterized by $J \times \operatorname{Pic}(J)$, which contains all trivial modifications of $W_{i}$. The remarkable result about this family is that usually it irredundantly yields all nearby deformations of each of its members. The exact statement is contained in

THEOREM 5. If $S$ is a nonhyperelliptic Riemann surface of genus $>2$, then the family $\mathcal{T}\left(\mathcal{W}_{i}\right)$ gives a complete family of deformations of each of its members. Furthermore the members of this family are all distinct.

The proof of this result uses correspondence techniques and may be found in [6]. In particular, the theorem shows that the space $H^{1}\left(J, \operatorname{Hom}\left(\mho_{i}, w_{i}\right)\right)$ of infinitesimal deformations of $\mathcal{Q S}_{i}$ has dimension $2 \mathrm{~g}$. This contrasts strongly with the classical case $g=1$ where this dimension is $g$. Furthermore, Gunning $[3,6]$ has shown that the space $H^{0}\left(J, \operatorname{Hom}\left(\mathscr{W}_{i}, \mathscr{W}_{i}\right)\right)$ is always one dimensional. Consequently the Picard sheaves $\mathcal{W}_{i}$ are indecomposable and not easily confused with themselves in strange ways.

Continuing with the assumption that $S$ is nonhyperelliptic of genus $>2$, I have been able to show some related results using correspondence methods on symmetric products [8].

THEOREM 6. (a) For any positive integer $n$, all deformations of the symmetric product $S^{(n)}$ are induced in a unique way by a deformation of $S$.

(b) The bundle $\int_{i t}: S^{(i)} \rightarrow J$ extends over a given deformation of $J$ if and only if the deformation of $J$ is the deformation of the Jacobian induced by $a$ deformation of $S$. In this case, the only possible such extensions are the obvious ones.

An interesting aspect of this last result is that it is related to the Schottky problem of characterizing Jacobians among all principally polarized Abelian varieties. In fact, in a neighborhood of our Jacobian $J$ in Siegel's upper half-plane, the locus $g$ of Jacobians is characterized by the existence of a lifting of the bundle $\int_{i t}: S^{(i)} \rightarrow J$ to $\mathscr{G}$. Thus a better understanding of Picard type bundles on Abelian varieties may throw some light on the classical problem of characterizing the Riemann matrices of Riemann surfaces.

REMARK 3. Recently in an unpublished work, X. Gomez-Mont and, also, S. Mukai have made contributions to these topics (especially in the direction of removing the above restrictions on the Riemann surface $S$ ).

5. An algebraic inversion problem. One might hope to say something significant about the Picard sheaves $\mho_{i}$ by using the methods of algebraic geometry. In general, any coherent sheaf on a projective variety may be 
described by a finitely generated graded module over a graded ring. In our case, the theta divisor $\theta$ (or a translate of it) is an ample divisor on the Jacobian $J$. Thus $J=\operatorname{Proj}(A)$ where $A$ is the graded ring $A \equiv$ $\bigoplus_{n \geqslant 0} \Gamma\left(J, \mathcal{O}_{i}(n \theta)\right)$. Furthermore $\mathcal{W}_{i}$ is the sheaf on $J$ associated to the graded $A$-module $M_{i}=\bigoplus_{n \geqslant 0} \Gamma\left(J, \mathscr{W}_{i}(n \theta)\right)$.

This leads one to ask for a solution of the following algebraic inversion.

Problem. Determine explicitly the structure of the $A$-modules $M_{i}$.

In the classical theory, the ring $A$ has been carefully studied. For instance, it is well known [14] that, for any positive integer $n$,

$$
\operatorname{dim}_{\mathbf{C}}\left(J, \vartheta_{J}(n \theta)\right)=n^{g} \text { and } H^{j}\left(J, \vartheta_{J}(n \theta)\right)=0 \text { if } j>0 .
$$

In [7], I have proven the analogous result for the module $M_{i}$ :

TheOREM 7. If $n(i-2 g+2)>g$,

$$
\operatorname{dim}_{\mathbf{C}} \Gamma\left(J, \mathscr{W}_{i}(n \theta)\right)=(i-g+1) n^{g}-g n^{g-1}
$$

and

$$
H^{j}\left(J, \mathscr{W}_{i}(n \theta)\right)=0 \text { for } j>0 .
$$

Much is known about the algebra of the rings $A$ [13]. One hopes to be able to understand the defining relations between generators of the modules $M_{i}$. Using Mumford's "Castelnuovo type" techniques [15] together with Theorem 7 , one can give some bounds on the degrees for generators and relations for the modules $M_{i}$, but the above inversion problem asks for more details than are presently known.

Lastly, I want to mention informally another result. Using correspondence techniques in [7], I have given isomorphisms between the graded components $\Gamma\left(J, \mathscr{W}_{i}(n \theta)\right)$ of the modules $M_{i}$ and the space of sections of some definite sheaves over Abelian coverings of $S$. I hope this isomorphism will help lead to a solution of the algebraic inversion problem.

\section{REFERENCES}

1. A. Grothendieck, Technique de descente et théorèms en géométrie algébraique. V. Les schémes de Picard: Théorèms d'existence, Séminaire Bourbaki, exposé 232 (1961-1962).

2. R. C. Gunning, Some special complex vector bundles over Jacobian varieties, Invent. Math. 22 (1973), 187-210.

3. _ Riemann surfaces and generalized theta functions, Ergebnisse der Math. und ihrer Grenzgebiete, Bd. 91, Springer-Verlag, Berlin, 1976.

4. G. Kempf, Some vector bundles on Jacobian varieties, Proc. Amer. Math. Soc. 54 (1976), 179-180.

5. A property of the periods of a Prym differential, Proc. Amer. Math. Soc. 54 (1976), $181-184$.

6. __ Toward the inversion of abelian integrals. I, Ann. of Math. (2) 110 (1979), 243-273.

7. __ Toward the inversion of abelian integrals. II, Amer. J. Math. 101 (1979), 184-202.

8 . Deformations of symmetric products, Riemann surfaces and related topics, Proc. 1978 Stony Brook Conference, Ann. of Math. Studies, Princeton, 1981.

9. I. G. Macdonald, Symmetric products of an algebraic curve, Topology 1 (1962), 319-343.

10. A. Mattuck, Symmetric products and Jacobians, Amer. J. Math. 83 (1961), 189-206.

11. Secant bundles on symmetric products, Amer. J. Math. 87 (1965), 779-797. 
12. D. Mumford, Lectures on curves on an algebraic surface, Princeton Univ. Press, Princeton, N. J., 1966.

13. __ On the equations defining abelian varieties. I, Invent. Math. 1 (1966), 287-354.

14. __ Abelian varieties, Oxford Univ. Press, Oxford, 1970.

15. - Varieties defined by quadratic equations, Questions on Algebraic Varieties, Centro Int. Math. Estivo, Roma, 1970.

16. R. L. E. Schwarzenberger, Jacobians and symmetric products. III, J. Math. 7 (1963), 257-268.

Department of Mathematics, The Johns Hopkins University, Baltimore, Maryland 21218 